Cahiers d'études italiennes

Enea Silvio Piccolomini-Pie II

\title{
La lettre à Mehmet II ou le loup et l'agneau
}

\section{Marie Viallon}

\section{OpenEdition}

\section{Journals}

Édition électronique

URL : http://journals.openedition.org/cei/81

DOI : $10.4000 /$ cei. 81

ISSN : 2260-779X

\section{Éditeur}

UGA Éditions/Université Grenoble Alpes

Édition imprimée

Date de publication : 15 octobre 2011

Pagination : 129-139

ISBN : 978-2-84310-207-3

ISSN : $1770-9571$

Référence électronique

Marie Viallon, « La lettre à Mehmet II ou le loup et l'agneau », Cahiers d'études italiennes [En ligne], 13 |

2011, mis en ligne le 15 avril 2013, consulté le 26 mars 2021. URL : http://journals.openedition.org/ cei/81 ; DOI : https://doi.org/10.4000/cei.81 


\title{
LA LETTRE À MEHMET II OU LE LOUP ET L'AGNEAU ${ }^{\text {I }}$
}

\author{
Marie Viallon \\ Université de Lyon 3
}

La lettre de Pie II, pape, à Mehmet II, sultan, est un unicum dans l'Histoire des relations entre l'Occident chrétien et l'Orient musulman, bien que les éléments extra-ordinaires, hors de l'ordinaire, n'y manquent pas; que l'on pense à la geste de Roland de Roncevaux, à la personnalité d'un Frédéric II de Hohenstaufen (I272-I337) ou à l'aventure du prince Djem qui a laissé sa marque jusque dans notre Creuse profonde ${ }^{2}$.

Ce texte, écrit pendant la retraite de Pie II à Sienne (entre le 3I janvier et le Io septembre I460), a été l'objet de toutes sortes d'interprétations et d'analyses et, pour ma part, j'ai déjà commis un premier travail ${ }^{3}$ où j'analysais comment Pie II rédige son texte pour signifier aux États et aux princes chrétiens, qui veulent commettre l'irréparable alliance avec les Turcs, que seul le souverain pontife a le droit et le pouvoir de remettre la couronne impériale. Constantin a eu son Sylvestre ${ }^{\text {er }}$, Charlemagne a eu son Léon III et Mehmet pourrait avoir son Pie II. C'est cette réaffirmation du pouvoir politico-temporel du pape sur les chrétiens qui résout l'apparente contradiction entre les tentatives réitérées par Pie II de prêcher la croisade anti-ottomane et sa proposition du trône impérial chrétien au sultan ottoman : en effet, dans les deux cas, le pape Pie II reste l'unique agent, il est caput et princeps mundi.

I. Ce sous-titre nous a été soufflé par un extrait d'une lettre de Nicolas Bessarion à son frère, le cardinal Jean Bessarion, à l'occasion de la chute de Négropont : Niun amore può essere tra pecore e lupi. Nicolas Bessarion, Epistole et orationes, Venezia, Valdarfer, I47I, f. $9 \mathrm{v}$.

2. À la mort de Mehmet II, le 3 mai I48I, la guerre fratricide entre ses deux fils, Bajazet et Djem, pousse ce dernier à se placer sous la protection des Chevaliers de Rhodes qui le vendent au roi de France; celui-ci l'exile à Bourganeuf.

3. Marie Viallon, "L'epistola latina a Maometto II », dans Luisa Secchi Tarugi (dir.), Pio II umanista europeo (Atti del XVII Convegno internazionale, Chianciano-Pienza, I8-2I luglio 2005), Firenze, Franco Cesati editore, 2007, p. 165-I77. 
Sans renier l'hypothèse précédente, je voudrais aujourd'hui tenter une autre voie interprétative en partant d'une remise en situation historique du texte étudié. En effet, cet homme connu pour son long combat en faveur de la croisade anti-ottomane - qui remplit des pages de ses Mémoires ${ }^{4}-\mathrm{a}$ été lu pape le 27 septembre 1458 et, un an plus tard, il rédige ce texte déroutant qu'il passe sous silence dans ses mêmes mémoires. C'est-à-dire qu'il change radicalement ses positions à propos du rapport conflictuel entre les chrétiens d'Occident et les musulmans d'Orient : quels sont les éléments de jugements qui nous permettent de comprendre ce revirement?

\section{La lettre}

Dans un premier temps, il convient d'examiner de plus près cette lettre en la parcourant à l'aune de ce qui nous intéresse et sans prétendre à une analyse complète. Ce document rédigé en latin comprend une cinquantaine de pages, nombre variable selon les éditions, et le manuscrit original est conservé à la bibliothèque apostolique du Vatican ${ }^{5}$; il a connu de nombreuses éditions incunables ${ }^{6}$, une moindre fortune dès le début du $\mathrm{XVI}^{\mathrm{e}}$ siècle ${ }^{7}$ et, enfin, un renouveau aux XX-XXI ${ }^{\mathrm{e}}$ siècles ${ }^{8}$.

Pie II ouvre sa lettre par le constat des nombreux conflits qui ont opposé les musulmans aux chrétiens :

Toi et tes ancêtres avez eu de nombreux conflits avec les chrétiens. Beaucoup de sang a coulé, bien des villes ont été détruites, des lieux saints incendiés, des vierges enlevées, des femmes violées, des champs dévastés. Tous les crimes imaginables ont été commis dans cette lutte pour l'empire que le Turc et le chrétien se disputaient, le glaive à la main?.

4. Enea Silvio Piccolomini [Pie II], I commentari, a cura di Mino Marchetti, Siena, Cantagalli, 1997, 2 vol. Dans la traduction française : Mémoires d'un pape de la Renaissance. Les "Commentarii " de Pie II, présentés et annotés par Ivan Cloulas, Paris, Tallandier, $200 \mathrm{I}$.

5. Cod. Vat. Lat. 7082.

6. On peut citer : Coloniæ, [s.n.], I464; Cologne, Ulrich Zel, I469; Treviso, Gerardus de Lisa, I475; Roma, Bartholomaeus Guldinkech, I477; Roma, Eucharius Silber, I485; Roma, Stephan Plamnck, I488. Ces trois dernières éditions accompagnées d'une Epistola Mahumetis ad Pium II.

7. On peut toutefois citer l'édition Coloniæ, Eucherij Cervicorni, I532.

8. La traduction italienne de Giuseppe Toffanin, Napoli, Pironti, I953; la traduction anglaise A. R. Baca, New York-Frankfurt, Peter Lang, 1989; la traduction française Anne Duprat, Lettre à Mahomet II, Paris, PayotRivages, 2002.

9. Luca D'Ascia, Il corano e la tiara. L'epistola a Maometto II di Enae Silvio Piccolomini, Bologna, Pendragon, 200I, p. 233 : "Multa tibi et tuis progenitoribus cum Christianis vella fuerunt, multus sanguis effusus est, multe urbes delete, sacrae ades incense, rapte virgines, violate matrone, vastati agri, scelerata perpetrata sunt quacumque in mentem venire possunt, dum Turca et Christianus, uter imperet, gladio contendit. " Trad. Duprat, ouvr. cité, p. 24 . 
D’entrée de jeu, il fait également le constat que la conquête ottomane est totale sur l'ensemble de l'empire d'Orient puisque, récemment, Mehmet II s'est emparé des deux ultimes bastions chrétiens de Sinope et Trébizonde. La chute de l'empire d'Orient est désormais une réalité :

Certes, tes ancêtres ont fait de grandes choses, et tes exploits ne le cèdent en rien aux leurs. Tu as pris Constantinople, enlevé aux Génois leur colonie de Péra, soumis la plus grande partie du Péloponnèse; en Rastie et en Valachie, tu as fait de belles conquêtes, vaincu bien des ennemis. Cette année encore, l'antique ville de Sinople, patrie de Mithridate Eupator, est tombée dans tes mains, avec son roi; tu as détruit Trébizonde et réduit en captivité ses habitants et son empereur. Enfin tu as vaincu Jean Cassano, qui avait osé te résister. Tu vois dans tout cela de grands exploits et nous t'accordons qu'ils ne sont pas des moindres ${ }^{\text {Io }}$.

En outre, il est de notoriété publique que le sultan ottoman - comme tous ses prédécesseurs - n'entend pas se contenter de l'Orient et qu'il convoite aussi l'Occident et, en particulier, Rome qui est nommée la pomme d'or dans les textes ottomans ${ }^{\text {II. }}$. Mais, dans la continuité de son combat pour la croisade anti-ottomane, le pape souligne la bien plus grande capacité des occidentaux à résister sur le plan militaire car ils sont animés spirituellement et moralement par le souffle d'une union religieuse autour de l'Église romaine, à la différence des chrétiens d'Orient divisés en de nombreuses Églises. La suite de la conquête ne s'annoncerait pas aussi facile que la prise de Constantinople qui était un fruit déjà blet.

Dès le second paragraphe de sa lettre, Pie II avance la proposition de conversion :

Donc, pour en venir enfin à l'objet de notre discours, te dire ce qui nous amène à t'écrire ceci, et te montrer le chemin de ta gloire et de ton salut, écoute avec attention ce qui suit. Si tu veux étendre ton empire aux peuples chrétiens et rendre ton nom glorieux entre tous, tu n'as pas besoin d'or, ni d'armes, ni de troupes, ni de vaisseaux. Une petite chose suffirait à faire de toi le plus grand, le plus puissant et le plus illustre des hommes qui vivent aujourd'hui. Tu veux savoir laquelle? Elle n'est pas difficile à découvrir et tu n'auras pas à la chercher bien loin car on la trouve partout : quelques gouttes d'eau pour te baptiser, t'initier au rite chrétien et à la foi dans l'Évangile. Si tu fais cela, il n'y aura plus de prince sur terre qui puisse te surpasser en gloire ou t'égaler en puissance. Nous t'appellerons empereur de Grèce et d'Orient et ces terres, dont tu

Io. D’Ascia, ouvr. cité, p. 234 : "Fatemur : res claras maiores tui egere, nec tua minora sunt opera, qui Constantinopolim expugnasti et Peram e regione Genuensium coloniam et Peloponnesum magna ex parte in deditionem accepisti, et in Rastia et in Valachia non parum agri adeptus es et saepe tuos hostes fudisti; et hoc anno Synopem vetustam urbem, Mithridati Eupatoris patriam, et eius tyrannum cepisti et, Trapezunte direpta, incolas eius et imperatorem in captivitatem abduxisti; et Johannem Cassanum, proelio congredi ausum, superasti. Magna haec tibi videntur, nec nos parva dicimus. " Trad. Duprat, ouvr. cité, p. 26-27.

II. Cette pomme d'or ferait référence au globe d'or que les représentations des empereurs byzantins tenaient en signe de pouvoir sur l'empire. Rome étant la cité des empereurs, elle est ainsi nommée. 
t'es emparé par la force et que tu détiens aujourd'hui sans aucun droit, deviendront alors ta propriété légitime ${ }^{12}$.

Il propose donc au sultan de conserver tous les territoires d'Orient conquis par les armes, voire d'agrandir son autorité sur les territoires d'Occident (et c'est par cette menace voilée que l'on sent que ce texte s'adresse aux princes occidentaux), en échange de quelques gouttes d'eau baptismale. À la suite de quoi, il dresse le tableau idyllique du nouveau monde créé par cette conversion.

Mais Pie II n'est pas né de la dernière goutte de pluie et il sait prévenir le possible retournement de proposition par lequel le même monde idyllique, pacifique et unitaire, pourra être institué par Mehmet II quand il soumettra tout l'Occident à l'Islam :

Mais, dira-t-on, cette paix, cette union, cette joie universelles peuvent exister sous ta Loi ${ }^{13}$.

Et Pie II introduit là une longue réfutation dogmatique de l'Islam qu'il voit comme une religion de l'épée et de la guerre, alors que le christianisme est une religion de l'amour et de la paix. Par là, il espère encore être entendu des turcophiles qui osent jouer l'impium foedum contre la puissance temporelle du pape.

En fin juriste et parfait avocat de sa cause, Pie II s'attache également à étudier l'hypothèse d'une éventuelle résistance des sujets ottomans à suivre leur maître dans cette conversion et il argumente du fait que nombre d'entre eux étaient auparavant des chrétiens, convertis de force à l'Islam, puis il avance les exemples de nombreux chefs barbares qui ont entrainé leurs peuples ad majorem Dei gloriam et pour leur plus grand bénéfice politique : Constantin le Grand, Clovis le Franc, Théodoric l'Ostrogoth, Agilulphe le Longobard...

À partir du cinquième paragraphe, Pie II passe du plan temporel au plan spirituel et développe sa réfutation de l'Islam à grand renfort de citations bibliques confrontées à des citations des sourates du Coran. Nous

I2. D'Ascia, ouvr. cité, p. 236-237: "Quocirca, ut eo tandem veniamus quo nostras festinat oratio et id dicamus quod ad scribendum compulit tuamque gloria et tuam salutem tibi denique ostendamus, abhibe his paucis animum. Si vis inter Christianos tuum imperium propagare et nomen tuum quam gloriosum efficere, non auro, non armis, non exercitibus, non classibus opus est. Parva res omnium qui hodie vivunt maximum et potentissimum et clarissimum te reddere potest. Quaris qua sit? Non est inventu difficilis, neque procul quarenda, ubique gentium reperitur : idest aqua pauxillum, quo baptezeris et ad Christianorum sacra te conferas et credas Evangelio. Hac si feceris, non erit in orbe princeps qui te gloria superet aut equare potentia valeat. Nos te Grecorum et Orientis imperatorem appellabimus et quod modo vi occupas et cum iniuria tenes possidebis iure. "Trad. Duprat, ouvr. cité, p. 32.

13. D'Ascia, ouvr. cité, p. 238 : "Dicet aliquis hanc pacem, hanc unionem, hanc communem latitiam haberi posse sub tua lege. " Trad. Duprat, ouvr. cité, p. 35. 
savons que Pie II n'avait pas de connaissance directe du texte du Coran mais il travaille à partir de compilations dressées par le cardinal Juan de Torquemada ${ }^{14}$, son ami Nicolas de Cuse ${ }^{\mathrm{Is}}$ ou le frère dominicain Ricoldo da Montecroce ${ }^{16}$. L'intention du pape est de démontrer que la doctrine musulmane ne résiste pas à la comparaison avec le christianisme.

En conclusion, dans le vingt-quatrième et dernier paragraphe, Pie II répète sa proposition :

Mais avant tout cela, nous t'avons montré que tu ne saurais obtenir auprès des chrétiens la gloire et la puissance auxquelles tu aspires manifestement, surtout parmi les nations d'Europe et d'Occident, tant que tu demeureras fidèle à ta secte. Si tu acceptais de recevoir les sacrements chrétiens, nous t’avons montré que tu pouvais espérer cette puissance et cette gloire ${ }^{17}$.

$\mathrm{Au}$ terme de cette lecture rapide, il est désormais acquis que ce texte est destiné à faire peur aux princes occidentaux tentés par une alliance avec les Turcs et qu'il n'a jamais été envoyé à Constantinople. D'ailleurs, les archives ottomanes n'en conservent aucun exemplaire, ni original ou copie en latin, ni traduction en grec selon les usages de la chancellerie ottomane, jusqu'en I49O. Mais pourquoi avoir choisi cette fable de la lettre familière à Mehmet II au lieu d'une admonestation directe des princes turcophiles? Ou bien, pourquoi ne pas user des armes de l'excommunication et de l'Interdit que les souverains pontifes savent parfaitement fulminer pour défendre leur pouvoir temporel face aux États modernes?

Pour trouver une réponse satisfaisante à cette question, il convient de ne pas oublier que Pie II n'a pas des réactions d'homme d'Église mais plutôt d'homme de lettres. L'humaniste Piccolomini sait pertinemment manier le genre littéraire de la lettre familière - fût-elle fictive! - qu'elle soit d'inspiration classique par Cicéron ou d'origine plus italienne par Pétrarque. Par ailleurs, le comédiographe Piccolomini sait camper une situation, nouer une intrigue, développer les argumentaires pour parvenir à un épilogue satisfaisant. Aussi, peut-on émettre l'hypothèse que l'idée de la fausse lettre lui aurait été soufflée par un courant d'opinion qui banaliserait le côté invraisemblable, voire choquant, de sa proposition.

I4. Juan de Torquemada (I388-I468) OP, Collectio toletana.

I5. Nicolas de Cuse, Confutatio legis late Saracenis a maledicto Mahumeto, Basileæ, Oporinus, I543, 2 vol.

I6. Ricoldo Pennini dit da Montecroce OP (I242-I320), Confutatio Alchorani qui est amplement diffusé dans le monde grec et est traduit en allemand par Luther (Wittenberg, I542). Sa critique de l'Islam doit beaucoup à son pèlerinage en Terre Sainte.

17. D’Ascia, ouvr. cité, p. 285 : "Ante omnia vero monstratum est non posse te assequi inter Christianos gloria et potentiam, quam videris optare, maxime apud europeo et occidentales populos, dum tua in secta perseveraris. Quodsi velles christianis initiari sacris, magnam tibi spem fecimus et potentia et gloria. " Trad. Duprat, ouvr. cité, p. 156. 


\section{L'avis des princes}

Passée la stupeur et le choc de la chute de Constantinople (le 29 mai I453) sous les coups de l'artillerie lourde et après cinquante-cinq jours de siège, le nouveau maître de Constantinople - Le Grand Saigneur selon Anatole France - a su conserver la plus grande part de l'héritage byzantin et, en particulier, il a intégré dans sa bureaucratie un grand nombre de personnalités non turques, originaires des territoires de l'empire ottoman. Par exemple, la chancellerie de Mehmet II est la chancellerie byzantine qui a d'ailleurs conservé le grec comme langue officielle : il faudra attendre I490, c'est-à-dire une génération, pour qu'une réforme introduise véritablement l'ottoman comme langue bureaucratique de l'administration.

Constantinople, érigée en capitale de l'empire ottoman, est restaurée, agrandie et repeuplée et le monde assiste à une transmission relativement pacifique de l'autorité et des pouvoirs; c'est une nouvelle étape de cette translatio imperii et nombreux sont les témoignages qui fondent le passage de l'imperium des mains des Césars byzantins vers celles des sultans. Le premier à en être convaincu est Mehmet II lui-même qui aime à porter l'aigle bicéphale du basileus brodé sur ses cafetans et qui a fait graver au-dessus de la porte de son palais de Topkapi la mention suivante :

Le Sultan des deux continents et l'Empereur des deux mers, l'Ombre de Dieu dans ce monde et dans l'autre, le Favori de Dieu sur les deux horizons, le Monarque de l'orbe terraqué, le Conquérant du château de Constantinople, le Père de la conquête, le Sultan Mehmet Khan, fils du Sultan Murad Khan, puisse Dieu rendre son empire éternel et exalter sa résidence au-dessus des étoiles les plus brillantes du firmament ${ }^{18}$ !

Cette inscription ne laisse la place à aucun doute sur la revendication d'une puissance absolue et incontestable.

Dans le camp chrétien, les exemples ne manquent pas de ceux qui sont enthousiastes à l'arrivée d'un jeune souverain puissant; il faut dire que la situation politique - et donc économique - à Constantinople était pathétique. Le Crétois Georges de Trébizonde (I395-I484), secrétaire pontifical, écrit, dans une oratio au sultan, qu'à Rome :

[...] personne ne doute qu'il fût de droit empereur des Romains [...] [car] empereur est celui qui à juste titre possède le siège de l'empire et le siège de l'empire romain est Constantinople. Donc, qui possède Constantinople, est empereur. Mais ce n'est pas des hommes mais bien de Dieu, par l'intermédiaire de ton épée, que tu tires ta possession

I8. Inscription placée au-dessus de la porte de Topkapi Sarayi à Istanbul. Traduction française de Paul Chemla, dans Robert Mansel, Constantinople. La ville que désirait le monde, Paris, Seuil, 1997. 
du trône. Donc, tu es le légitime empereur des Romains! Celui qui continue à être empereur des Romains est aussi empereur de tout le globe ${ }^{19}$ !

De la même manière, la République de Venise, signant rapidement un nouveau traité de paix et de commerce, reconnaît de facto la souveraineté du sultan sur tous les territoires byzantins, y compris les villes des Pouilles : Brindisi, Lecce, Tarante et Otrante.

Par un autre moyen, Laurent le Magnifique proclame la même certitude. Il fait graver par Bertoldo di Giovanni (I44O-I49I) une médaille qui porte au recto, autour du profil du sultan conquérant, l'inscription :

Mahumet, Asie ac Trapesunzis Magneque Gretie Imperator,

et, au verso, figure un triomphe où le sultan est conduit par Mars et suivi par les terres conquises: Grèce, Trébizonde et l'Asie mineure ${ }^{20}$.

Pour sa part, Ferrante d'Aragon fait frapper deux médailles avec le profil du sultan et ces deux inscriptions :

Asie et Gretie imperator

et

Bizantii imperator.

Le terme d'empereur/imperator (c'est nous qui soulignons par l'italique) est attribué à Mehmet II sans barguigner : on reconnaît alors l'imperium militiae commandement militaire du sultan autant que l'imperium domi, c'est-à-dire le pouvoir civil d'énoncer le droit et de rendre la justice, d'organiser la politique et de garantir l'ordre civil. Dans la suite logique de l'empire de Constantin le Grand et suivant la Loi de la doctrine musulmane, Mehmet II est ombre de Dieu sur terre, il réunit la puissance politique et les pouvoirs religieux.

La proposition pontificale d'ériger Mehmet II en empereur des Grecs et de l'Orient n'a donc rien d'original dans ces années qui suivent la chute de Constantinople. Toutefois, Pie II va plus loin que ses contemporains puisqu'il ne se contente pas de se soumettre à la force des faits et d'entériner l'inévitable, avec une grande sagesse et une parfaite clairvoyance politique. Le pape propose le baptême, c'est-à-dire qu'il se propose d'accueillir Mehmet II dans le cercle des souverains chrétiens.

Quel est l'élément de la personnalité ou de la biographie du sultan qui autorise le souverain pontife à oser une telle proposition?

19. A. Mercati, «Le due lettere di Giorgio da Trebisonda a Maometto II », dans Orientalia christiana periodica, IX (1943), p. 65-99.

20. Un exemplaire en a été vendu à Londres, le 23 février 2004, pour la somme de 3600 livres. 


\section{La foi (?) de Mehmet II}

À la fin du $\mathrm{xv}^{\mathrm{e}}$ siècle, Mehmet II a été l'objet de très nombreux portraits rédigés par des auteurs très différents par leurs âges, leurs statuts sociaux et leurs origines; ils insistent tous sur son courage et sa cruauté au combat, sur son intelligence, sur son goût pour les arts ${ }^{21}$ et les lettres ${ }^{22}$, sans négliger les arts mécaniques ${ }^{23}$; ils mettent l'accent sur son ambition qui le fait rêver de gloire éternelle et de puissance incomparable.

Presque tous les descripteurs évoquent la question de sa foi, comme si elle n'allait pas de soi. Ainsi, Giovanni Maria Angiolello, originaire de Vicence, qui est entré au service du sultan en I474, écrit-il :

E disse il detto Bayasit che suo padre era padrone e che non credeva in Maccometto. Ed in effetto, era così, per quello dicono tutti questo Mehemet non credeva in fede alcuna $^{24}$.

On peut citer d'autres auteurs comme Paolo Giovio et Francesco Sansovino qui ne sont pas des contemporains du sultan mais qui se nourrissent des mêmes sources de chroniqueurs, d'ambassadeurs, de mercenaires, d'aventuriers et d'autres voyageurs du temps de la conquête.

Paolo Giovio - qui a pris ses renseignements dans l'Historia turchesca d'Angiolello, dans l'Oratio à Innocent VIII de Filippo Buonacorsi ${ }^{25}$, dans

2I. Pour ne citer que lui, Giorgio Vasari rappelle que - bien que l'Islam interdise les images - Mehmet II a sollicité le Sénat vénitien pour qu’il lui envoie son meilleur portraitiste : Gentile Bellini est parti le 3 septembre I479 pour Constantinople où il a réalisé le fameux portrait de Mehmet II, désormais conservé à Londres, ainsi que de nombreux autres portraits de courtisans ottomans et une ample veduta de Venise, à fresque.

22. André Clot, Mehmet II. Le conquérant de Byzance, Paris, Perrin, 1990, p. I53. Il donne un exemple d'une de ses compositions de gazel ou rhazal [ces courts poèmes inspirés de la poésie amoureuse persane où le nom du poète doit figurer au dernier vers] dédiée à un jeune religieux chrétien de Galata : "J'ai vu un ange, au visage de soleil, qui est la lune de l'univers. / Les noires jacinthes de ses cheveux bouclés sont le soupir des amants / Il est comme une lune éclatante habillée de noir, ce gracieux cyprès, / Il est bien, par sa beauté, le roi du pays des Francs. / Quiconque n'attache pas son cœur au nœud de sa ceinture monastique / N'est pas homme de foi, mais le plus égaré des amants. / À ceux que tuent ses œillades, ses lèvres redonnent la vie, / Bien que ce donneur d'âme ait pour voie la religion de Jésus. / O Avni! Ne doute pas qu’il t’obéisse, ce charmant! / Toi, tu es roi d'Istambul et lui, celui de Galata. "

23. Giovanni Maria Angiolello (I45I-I525), Il sultano e il profeta: memorie di uno schiavo vicentino divenuto tesoriere di Maometto II il Conquistatore, Milano, Serra e Riva, 1983, p. I53 : "Il detto sigor Mehemet imparò a lavorare diverse cose, e continuamente lavorava come anelli da arco, cavi da centure e vagine da Spada, e pizachi (?) e questo faceva per passar il tempo. " - Angiolello, capturé à Négrepont en I470, entre au service des Ottomans et devient trésorier du sultan. À ce titre, il participe aux campagnes militaires de I476-I477 en Moldavie et en Hongrie puis à celle du Caire en I48I. Pendant le règne de Bajazet, il part en Perse. En 15I4, il rentre en Italie et, dès I5I7, il préside le collège des notaires de Vicence, jusqu’à sa mort en I525.

24. Angiolello, ouvr. cité, p. I53.

25. Filippo Buonacorsi (I437-I496), Ad Innocentium VIII de bello turcis inferendo oratio, Warzarwa, Panstwowe Wydawnictwo Naukome, 1964 . 
le traité de l'évêque Stefano Taleazzi ${ }^{26}$ et dans des récits oraux de soldats et d'ambassadeurs - raconte :

Molti estimorono che non credessi più nella fede di Maometto che in quella di Cristo e de' Gentili, per essere allevato in infanzia da sua matre qual fu figliuola del dispoto Lazaro di Servia, e teneva la fede cristiana e gli imparava l'Avemaria e il Paternostro, ma poi che fu adulto e retirandosi alla fede maomettana, si portò di sorte che non tenne né l'una né l'altra per il che non mantenea la parola se non quanto gli venea bene, e nulla cosa stimava essere peccato per adempire gli appetiti $\operatorname{suoi}^{27}$.

Il porte crédit à l'idée que la mère de Mehmet II, Huma Hatun, étant fille du Despote Lazare de Serbie, l'aurait élevé dans la religion chrétienne qu'elle a toujours pratiquée et qu'elle lui aurait enseigné les prières fondamentales de la religion chrétienne.

Pour sa part, Francesco Sansovino fait sur la foi de Mehmet II une observation singulière selon laquelle il estimerait que la religion convient plutôt aux marchands qu'aux princes car les premiers vivent du crédit que l'on a en leur foi, alors que les princes s'imposent par la force de leurs armes.

À ces quelques témoignages qui sèment le doute sur la sincérité et la solidité de la foi musulmane de Mehmet II, s'ajoutent quelques remarques qui ont certainement augmenté le trouble chez ses contemporains et on se contentera, ici, de n'en citer que trois. Ainsi, Jacopo Tebaldi dans sa description de la chute de Constantinople souligne-t-il que Mehmet II laisse ses nouveaux sujets parfaitement libres de pratiquer le culte chrétien ${ }^{28}$ et l'on sait qu'il a, peu après la conquête, convoqué le patriarche de Constantinople, Gennadios Scholarios, pour qu'il l'éclaire sur le dogme chrétien ${ }^{29}$. En outre, cette conquête place entre les mains du sultan les reliques de la Passion du Christ (la tunique, des fragments de la croix, la couronne d'épines, la véronique...) auxquelles la doctrine musulmane n'accorde aucune valeur puisqu' elle estime que la crucifixion est une mystification, mais le sultan veille à les protéger du pillage et des destructions. Enfin, le franciscain vénitien, Francesco Suriano (I45O-I529), raconte ${ }^{30}$ que le sultan aurait reçu le peintre Gentile Bellini dans une pièce de son palais où brûlait une lampe à huile, placée devant une icône de la Vierge.

26. B. Feliciangeli, "Stefano Taleazzi, Declaratio generalis ex gestis maiorum et noviter deducti de modo servando in expeditione contra Turcos ", dans Archivio della R. società romana di storia patria, XL (1917), p. 6-63.

27. Paolo Giovio, Commentario de le cose de' Turchi, Venezia, Aldo Manuzio, I54I; Bologna, Clueb, 2005, p. $97-98$.

28. Agostino Petrusi, La caduta di Costantinopoli. Le testimonianze dei contemporanei, Milano, Mondadori, I986, p. I80 et suiv.

29. La discussion entre le sultan et le patriarche a été rapportée par l'interprète dans Le journal de Ebuzziya (Mecmua-i Ebuzziya) publié à Istanbul, en I9II.

30. Francesco Suriano, Il trattato di Terra Santa e dell'Oriente (I524), Milano, Girolamo Golubovich, I90o. 
Ces évocations rapides insinuent le doute : et si Mehmet II était plus chrétien qu'il n'y paraît? S'il s'en fallait de peu qu'il n'accepte le baptême? Cette hypothèse est brièvement évoquée par Francesco Filelfo (I398-I48I), un humaniste qui a été secrétaire de l'ambassade de Venise à Constantinople et qui écrit une ode au sultan :

Si le Christ, fils de Dieu le Très-Haut, qui est aussi Dieu le Père, t’avait donné à toi, Mehmed, l'œil de la foi, tu serais certainement le roi de toute la terre ${ }^{31}$.

Et Georges de Trébizonde, déjà évoqué, va plus loin encore dans le rêve et l'utopie pour sauver la foi, garantir la paix... et épargner à l'Europe une domination turque cruelle et violente. En effet, dans un traité épistolaire intitulé De la vérité de la foi des chrétiens, rédigé à Naples en juillet I453, il invite le Grand Turc à se convertir pour réaliser la fusion de l'Islam et du christianisme, pour convoquer un concile islamo-chrétien et pour créer un empire nouveau et puissant sous son gouvernement :

Se uno riuscisse ad unire in una sola fede ed in una sola confessione queste due stirpi, cioè quella cristiana e quella musulmana, io giuro per il Dio del cielo e della terra che costui sarà esaltato presso tutti gli uomini, e in cielo e in terra, e passerà nell'ordine degli angeli. Nessuno meglio di te, mirabile emiro, può portare a termine una impresa del genere. [Et il poursuit] Una tale e tanto grande vittoria concessa da Dio alla tua sublimità dimostra chiaramente che Dio vorrebbe che tu imitassi quel Costantino di cui ti concesse il trono e la città, e che tu investigassi come lui, i problemi riguardanti Cristo. Chiunque ricerchi, trova, dice Cristo stesso, e a colui che batta sarà aperto ${ }^{32}$.

Ce même Trébizonde a de la suite dans les idées car, en 1465 , il fait le voyage jusqu'à Constantinople en espérant obtenir une audience pour expliquer, en personne, son projet au sultan, mais en vain : l'audience ne lui est pas accordée. Il ne s'avoue pas vaincu et, en I467, il rédige un traité intitulé Au divin Manuel qui sera sous peu roi de l'univers dans lequel il affirme que le nom de Mehmet est une variante d'Emmanuel, élu par Dieu pour une mission d'unification politique et religieuse du monde.

\section{Conclusion}

On voit à travers ce rapide excursus dans les textes de l'époque que l'idée de Pie II de proposer quelques gouttes d'eau baptismale au sultan des Turcs n'est pas aussi surprenante - et farfelue - qu'il peut y paraître. Replacée

3I. Émile Legrand, Cent dix lettres grecques de Francesco Filelfo, Paris, Leroux, I892, p. 2 I4.

32. Cité dans Agostino Petrusi, La caduta di Costantinopoli, ouvr. cité, p. 77 et p. 79. 
dans son contexte, la Lettre à Mehmet II devient presque banale et admissible à tous ceux qu'anime le sens de la paix et de l'harmonie du monde. Il resterait à s'interroger sur la date de rédaction de cette lettre. Pourquoi attendre I460 pour tenter d'apporter un remède à une situation apparue dix-sept ans plus tôt?

Là encore, un texte de l'époque détient peut-être la clé. Il s'agit d'une prophétie, née certainement à Venise, qui annonce que le Turc devra conquérir Constantinople, qu'il poussera jusqu' au Danube, prendra Rome et persécutera les chrétiens, et alors - mais alors seulement - un homme juste et pieux apparaîtra à Venise pour conduire le sultan et son peuple à la conversion : le Turc occupera le trône impérial et l'homme pieux celui de saint Pierre : [...] et omnia ista complebuntur antequam perficientur anni Domini quadringentesimi sexagesimi primi. (" [...] et tout ceci s'accomplira avant que ne se termine l'an de Grâce I46I ${ }^{33}$. »)

Pie II s'est-il reconnu dans cet homme pieux? 
